QUALTFYING A MOSAIC OF DET'ECTORS

FOR

INFRARED ASTRONOMY

Observatoire de Meudon

F.Laccmbe, D.Rouan, D.Stefanovitch, B.Talureau :

Laboratoire d'Astronomie Infrarouge

J.Berezné, G.Epstein, S.Pau, D.Phan Van, Y.Zéau:

Département de Recherches Spatiales

\title{
I. IITTRODUCTION
}

A $8 \times 8$ InSb CID array, developed in France by SAT (1) (Société Anonyme de Télécommunications) for terrestrial applications, has been tested at Meudon Observatory in order to evaluate if its performances at LHe temperature, meet the requirements of infrared astronomy. Next sections give : 1) Description of the array and the experimental conditions; 2) The test results; 3) Work under development.

1) Description of the device and of the test procedures : the pixel's dimension is $165 \times 110$ sq.microns with a filling factor of 17\%. The infrared material is InSb sensitive up to 5.5 microns and the temperature the detector was designed to work at is 77K (IN2). The array consists of $8 \times 8$ pairs of MIS (Metal Insulator Semiconductor) capacitors, one of the pair being connected to a row line and the other to a column line. By means of scanners, different voltages can be applied to the lines, so that minority carriers (holes) are either trapped in the potential wells under the column. or transferred under the row electrode or injected in the substrate by recombination with the electrons. The reading consists: in the measurement of the variation of voltage at the end of one line when charges, accumulated during integration time, are transferred from row to column electrodes. Both the thermally generated and the photon-generated electron-holes pairs contribute to the accumulated charge. Decreasing the temperature has thus two advantages from the point of view of low flux level detection : a) the capacity of potential wells for photon-generated charges is increased, b) the integration time can be much larger and, since read noise is dominant, the signal to noise ratio can be largely improved.

A IHe cryostat was thus equipped with one mosaic in a housing with a hole providing a $\mathrm{f} / 5$ beam; a filter wheel equipped with $\mathrm{K}(2.2 \mu \mathrm{m}), \mathrm{L}(3.6 \mathrm{pm}), \mathrm{M}(4.7 \mathrm{pm})$ and CVF filters allows work at different wavelengths. Mounted on an optical bench, a black-body, with either large or small apertures and a Ge lense are used to feed the mosaic with flat field of with point sources at different temperatures. In a first phase, the electronics has been just modified in order to authorize integration times larger than the nominal $\mathrm{T}=100 \mathrm{\mu s}$ of. the original configuration at IN2 temperature. In a second phase new electronics has been developed in our laboratories and is more suitable in terms of large integration time, data acquisition and noise immunity. Note that the integration of the preamp stage inside the cryostat led, to a reduction by a factor of 3 of the electronic noise. In parallel, a data acquisition system, based on a Z80 $\mu$ P was developed. It features : voltages control of the read electronics, control of the integration time, data acquisition and storage, pre-processing (average, noise, substraction of background etc.), real time display of the infrared picture.

\section{TEST RESULTS}

Dark current. The time for a potential well to saturate with thermally generated charges has been studied with the cryostat totally closed, during cooling-down phases. The results for an elementary MIS capacitor and one nixel from a complete

Proceedings of the IAU Colloquium No. 79: "Very Large Telescopes, their Instrumentation and Programs", Garching, April 9-12, 1984. 
mosaic are shown in Fig.1. The main result is the possibility of integration time up to $10 \mathrm{sec}$ at LHe temperature

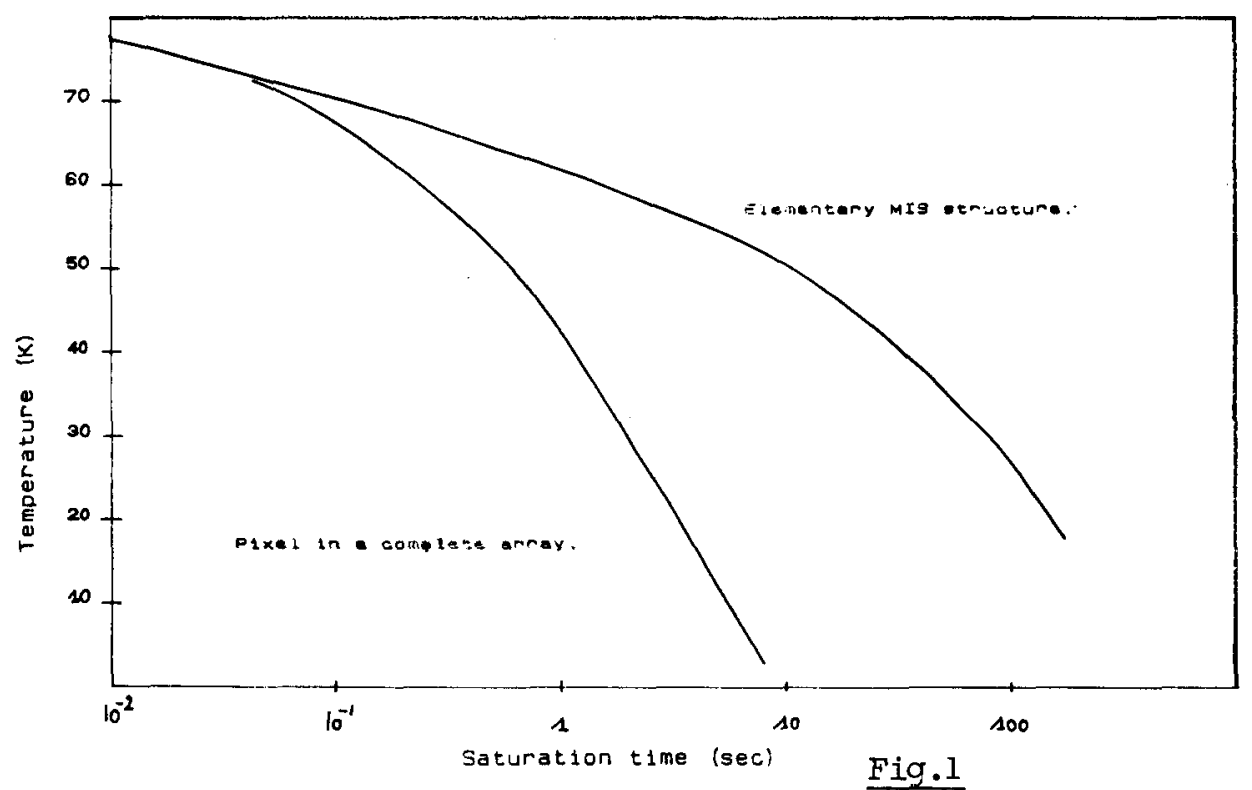

Linearity and storage capacity. Shown on Fig.2 is the response in number of electrons to an input flux of photons for respectively the LN2 and the LHe temperatures. The linearity appears to be good, even at low fluxes levels (no threshold effects). The maximum storable charge of 6 millions of electrons allows a comfortable dynamic range.

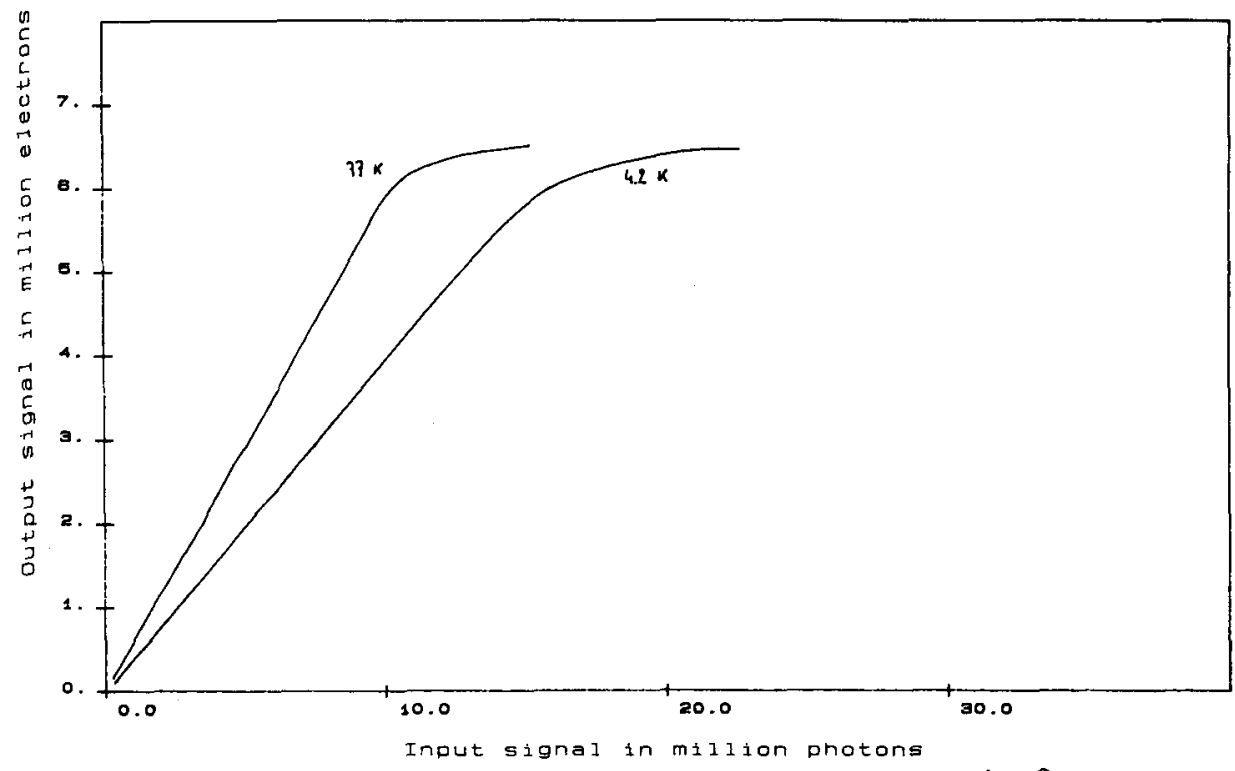

Fig. 2

Quantum efficiency. The quantum efficiency is measured with small holes in front of the black-body, so that the image of the hole (including diffraction) is smaller than the size of the sensitive area of the pixel. This procedure appears to be more precise than flat field measurements. The average quantum efficiencies at IN2 and LHe temperatures are respeetively $61 \%$ and $39 \%$, with a dispersion of $\pm 5 \%$. Recently a new mosaic from SAT has been tested revealing a much better homogeneity from pixel to pixel. 
Lack of time-lag effect. Response lag with time constants up to several seconds are reported (2) for low level fluxes. This point has been carefully examined and we found no effects of that kind at least at a background level as low as low as $50000 \mathrm{e}^{-}$. A likely interpretation is that the peculiar method used to read the . mosaic provides an almost complete emptying of the wells.

Noise : the best achieved read noise (preamp. inside the cryostat) is $1400 \mathrm{e}^{-}$ r.m.s., this means that with a $10 \mathrm{sec}$ integration time and a quantum efficiency of 408 , a NEP of $4.410^{-17} \mathrm{~W} \mathrm{~Hz}-1 / 2$ is reached at $5 \mathrm{pm}$ in absence of background radiation.

III. WORK UNDER PROGRESS.

Testing a new type of $8 \times 8$ array has been started recently. The main advantage with respect to the preceding type are the better filling factor (39\%) and the better homogeneity from pixel to pixel, the other characteristics being more or less identical. A $32 \times 32$ array is expected to be on loan in a very close future.

A first attempt for astronamical imaging has been recently undertaken, mounting the test photometer at the $\mathrm{f} / 5$ focus of the $2 \mathrm{~m}$ telescope of Pic du Midi Observatory. Bad weather conditions allowed only few hours of observation with, at any opportunity, a high hygrametry ( $100 \%$ under the dame) and a bad seeing due to a strong wind. Qualitatively good results have however been obtained and Fig.3 shows 2 examples of images obtained on a single star (Arcturus) and on a double system with 4 " separation ( $\boldsymbol{y}$ Vir).
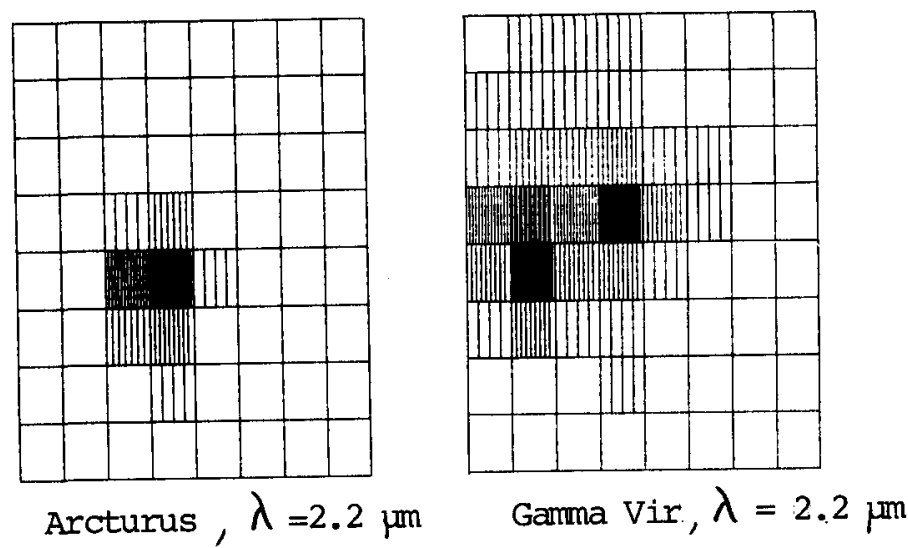

Fig.3

At $2.4 \mathrm{~m}$ integration time up to $2 \mathrm{sec}$ was currently achievable, but at longer wavelengths, in addition to the strong absorption, the water vapor was at the origin of a high background emission so that only short elementary integration time was possible ( $\mathrm{T}<32 \mathrm{~ms}$ ) .

The use of arrays of micro-lenses may be a way to overcome the problem of the filling factor as shown on Fig. 4. We are developing such arrays using fluored glass (3) (transparent up-to 8 m) moulded on a brass matrix. 


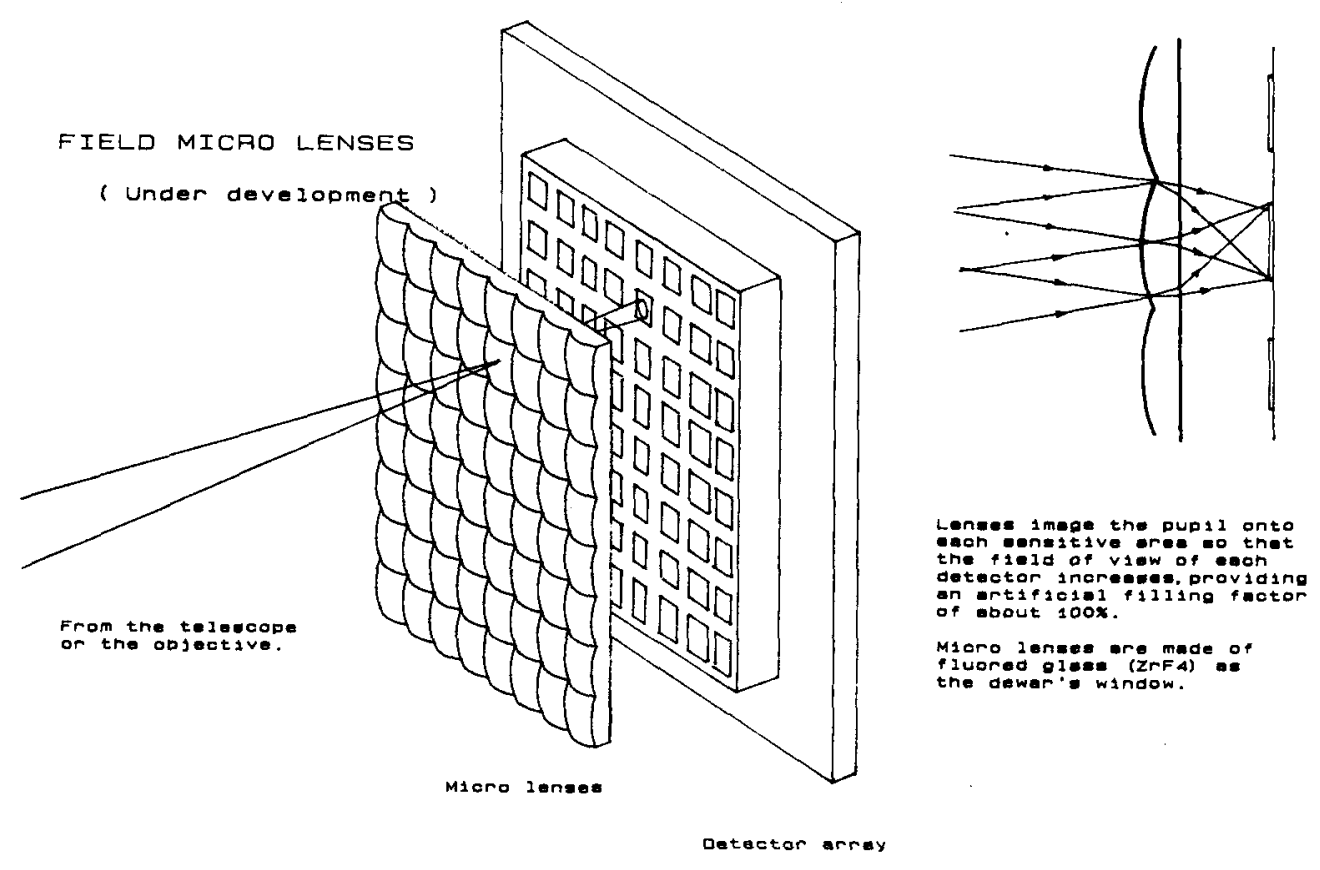

Fig. 4

\section{References}

(1) CHATARD J.P., et al. (1978). Revue de Physique Appliquée, 13, 637.

(2) FOWLER A.M. and BRITT J.P. (1982), SPIE 331, 18. Instrumentation in Astronomy IV.

(3) POUIAIN M. and LUCAS J. (1978), Verres Réfract. 32, 4. 